\title{
A SET OF POSTULATES FOR GENERAL PROJECTIVE GEOMETRY*
}

BY

\author{
MEYER G. GABA
}

Since Klein promulgated his famous Erlangen Programme $\dagger$ it has been known that the various types of geometry are such that each is characterized by a group of transformations. In view of the importance of the concept of transformation in nearly all mathematics and perhaps especially in geometry, geometers may properly seek to develop the various types of geometry in terms of point and transformation. For euclidean geometry this has been done by Pieri. $\ddagger$

This paper is devoted to a similar treatment of general projective geometry. $\S$ One would naturally lay such postulates on the system of transformations so as to make the system form the group associated with the geometry. This was the scheme that Pieri used. His postulates make his transformations form the group of motions. In general projective geometry, however, this method is not necessary. If we are given the group of all projective transformations we can deduce the geometry from it but it will be shown in the sequel that we can also do that from a properly chosen semi-group belonging to that group.

Our basis, to repeat, is a class of undefined elements called points and a class of undefined functions on point to point $\|$ or transformations called collineations. For notation we will use small Roman letters to designate

* Read before the American Mathematical Society, April 26, 1913.

$\dagger$ F. Klein, Vergleichende Betrachtungen über neuere geometrische Forschungen, Erlangen, 1872. English translation by M.W. Haskell, B u ll et in of the A meric a n M a the matic a l S o c i e ty, vol. 2 (1893).

$\ddagger$ M. Pieri, Della geometria elementare come sistema ipotetico-deduttivo; monografia del punto e del mote, Me morie delle Scienze di Torino (1899).

$\S$ General projective geometry is defined by Veblen and Young as a geometry associated (analytically) with a general number field; that is, its theorems are valid, not alone in the ordinary real and the ordinary complex projective spaces but also in the ordinary rational spaces and in the finite spaces. This paper connects closely with the postulates for general projective geometry given by $\mathrm{O}$. Veblen and $\mathrm{J}$. W. Young in the A meric a $\mathrm{n}$ J o urnal of M a t h e m a ti c s, vol. 30 (1908), and in their Projective Geometry, Ginn and Company (1910).

$\|$ By point to point is meant that to every point $p$ there corresponds a single point $p^{\prime}$ and no point $p^{\prime}$ is the correspondent of two distinct points $p_{1}$ and $p_{2}$. 
points and small Greek letters for collineations. Thus

$$
\tau\left(p_{1}, p_{2}, p_{3}\right)=p_{1}^{\prime}, p_{2}^{\prime}, p_{3}^{\prime}
$$

means that the collineation $\tau$ transforms the points $p_{1}, p_{2}, p_{3}$ into $p_{1}^{\prime}, p_{2}^{\prime}, p_{3}^{\prime}$ respectively. Line will be defined in terms of points and collineations. If we should interpret our undefined collineations as the group of all projective collineations, our defined line will satisfy the Veblen-Young assumptions for their undefined line and the postulates I to VIII that we will soon give are theorems in general projective geometry. This proves the consistency of our postulates. On the other hand, leaving collineation as undefined and using postulates I to $\mathrm{VI}^{*}$ we are able to prove as theorems the Veblen-Young assumptions $A_{1}, A_{2}, A_{3}, E_{0}, E_{1}, E_{2}, \cdots, E_{n}, E_{n^{\prime}}$, and $P . \dagger$ This shows that our six postulates are sufficient to establish the general projective geometry of $n$-dimensions. The undefined collineation will be proven to be a projective collineation which justifies the notation. If we desire that our class of collineations should be the group of all projective collineations we add postulate VII to the preceding six. To the Veblen-Young postulate $H_{0}$ corresponds our postulate VIII.

The independence of our assumptions is proven by the set of independence examples given at the end of this paper.

Postulate I. There are at least $n+2$ distinct points.

Postulate II. If $\tau_{1}$ is a collineation and $\tau_{2}$ is a collineation then the resultant of operating first with $\tau_{1}$ and then with $\tau_{2}$ (in notation $\tau_{2} \tau_{1}$ ) is also a collineation.

Definition. A linear set is a class of points such that:

(a) every collineation that leaves two distinct points of the class invariant leaves the class invariant,

(b) every collineation that leaves three distinct points of the class invariant leaves every point of the class invariant.

Definition. Points belonging to the same linear set are called collinear.

Definition. A linear set that contains at least three distinct points is called a line. $\ddagger$

Postulate III. If $p_{1}, p_{2}, p_{3}$ are three distinct collinear points and $p_{1}^{\prime}, p_{2}^{\prime}, p_{3}^{\prime}$ are three distinct collinear points then a collineation exists that transforms $p_{1}, p_{2}, p_{3}$ into $p_{1}^{\prime}, p_{2}^{\prime}, p_{3}^{\prime}$ respectively.

Postulate IV. If $p_{1}, p_{2}, p_{3}, p_{4}$ are four distinct points such that no three

* Postulates I to VI explicitly require that our set of collineations form a semi-group but all sets of transformations that we found satisfying I-VI were groups. The question whether they necessarily form a group in the general case has not as yet been proven.

$\dagger$ The precise statement of these postulates is given later in this paper.

$\ddagger$ It will be proved that every pair of distinct points is contained in one and only one line. 
are such that each is collinear with the same two distinct points then a collineation exists leaving $p_{1}$ and $p_{2}$ invariant and interchanging $p_{3}$ and $p_{4} .^{*}$

Definition. If $p_{1}, p_{2}$ are two distinct points, a line containing $p_{1}$ and $p_{2}$ or, in case no line exists containing $p_{1}$ and $p_{2}$, then the pair of points $p_{1}, p_{2}$ is called a one-space $\left(P_{1}\right)$ containing $p_{1}, p_{2}$.

Definition. If $P_{k-1}$ is a $(k-1)$-space and $p_{0}$ is a point not contained in $P_{k-1}$, the class $P_{k} \equiv\left[P_{k-1}, p_{0}\right]$ of all points $p$ collinear with the point $p_{0}$ and some point of $P_{k-1}$ is called the $k$-space determined by $P_{k-1}$ and $p_{0}$.

Definition. $k$ points are called independent of each other if there exists no $(k-2)$-space that contains them all.

Postulate V. If $p_{1}, p_{2}, \cdots, p_{n+1}$ are $n+1$ distinct points of the same $k$-space, $k<n$, then a collineation distinct from the identity exists leaving $p_{1}$, $p_{2}, \cdots, p_{n+1}$ invariant. $\dagger$

Postulate VI. If $p_{1}, p_{2}, \cdots, p_{n+2}$ are $n+2$ distinct points then there exists a $k$-space, $k \leq n$, that contains them all.

Postulate VII. If $p_{1}, p_{2}, \cdots, p_{n+2}$ are $n+2$ points of the same $n$-space such that every $n+1$ are independent, and $p_{1}^{\prime}, p_{2}^{\prime}, \cdots, p_{n+2}^{\prime}$ are $n+2$ points of the same $n$-space such that every $n+1$ are independent then a collineation exists that transforms $p_{1}, p_{2}, \cdots, p_{n+2}$ into $p_{1}^{\prime}, p_{2}^{\prime}, \cdots, p_{n+2}^{\prime}$ respectively.

Definition. A complete quadrangle is a figure consisting of four distinct coplanar points such that no three are collinear, called its vertices and six distinct lines containing the vertices in pairs called its sides. Two sides having no vertex in common are called opposite and points common to two opposite sides are called diagonal points.

Postulate VIII. The diagonal points of a complete quadrangle are noncollinear.

THEOREM 1. If $p_{1}$ and $p_{2}$ are distinct points, there is not more than one line containing both $p_{1}$ and $p_{2}$.

Let us assume that two lines $P$ and $P^{\prime}$ exist such that each contains $p_{1}$ and $p_{2}$. If the two lines are distinct then at least one of the lines must contain a point not in the other. Let us assume that $p_{3}^{\prime}$ is a point of $P^{\prime}$ and not of $P$. Since $P$ is a line it contains in addition to $p_{1}$ and $p_{2}$ a third point $p_{3}$. From Postulate III we know that a collineation $\tau$ exists such that $\tau\left(p_{1}, p_{2}, p_{3}\right)$ $=p_{1}, p_{2}, p_{3}{ }^{\prime}$. But $\tau$ leaves two points of $P$ invariant, therefore it leaves $P$ invariant and $p_{3}$ cannot be transformed into $p_{3}^{\prime}$ which is a point not of $P$. Since we are led to a contradiction our assumption that $p_{1}$ and $p_{2}$ are contained in two distinct lines must be false.

TheORem 2. Two distinct lines cannot have more than one common point.

\footnotetext{
* Compare Postulate IV with Theorem 4. Postulate IV is weaker than Theorem 4 as independence example - IV will show.

$\dagger$ Postulate $\mathrm{V}$ with Theorem 14 shows that all points lie in no $P_{k}, k<n$.
} 
Theorem 3. If three points are such that each is collinear with the same two points, they are collinear and conversely.

THEOREM 4. If $p_{1}, p_{2}, p_{3}, p_{4}$ are four distinct points such that no three are collinear then a collineation $\tau$ exists such that $\tau\left(p_{1}, p_{2}, p_{3}, p_{4}\right)=p_{1}, p_{2}, p_{4}, p_{3}$.

The theorem follows from Postulate IV and Theorem 3.

Theorem 5. If $p_{1}, p_{2}$ are two distinct points and $q_{1}, q_{2}$ are two distinct points then a collineation $\tau$ exists such that $\tau\left(p_{1}, p_{2}\right)=q_{1}, q_{2}$.

If $p_{1}, p_{2}$ are on a line and $q_{1}, q_{2}$ are on a line, the theorem follows from Postulate III. If no three of the points $p_{1}, p_{2}, q_{1}, q_{2}$ are collinear and they are all distinct then by Theorem 4 we know that a collineation $\tau_{1}$ exists such that $\tau_{1}\left(p_{1}, q_{1}, p_{2}, q_{2}\right)=p_{1}, q_{1}, q_{2}, p_{2}$ and a collineation $\tau_{2}$ exists such that $\tau_{2}\left(p_{1}, q_{1}, q_{2}, p_{2}\right)=q_{1}, p_{1}, q_{2}, p_{2}$. Therefore by Postulate II a collineation $\tau_{2} \tau_{1}$ exists such that $\tau_{2} \tau_{1}\left(p_{1}, p_{2}\right)=q_{1}, q_{2}$.

There are, notation apart, two possible cases remaining which are: (1) $q_{2}$ collinear with $p_{1}$ and $p_{2}$ but $q_{1}, q_{2}$ on no line; (2) $p_{2}$ collinear with $q_{1}$ and $q_{2}$, but $p_{1}, p_{2}$ on no line. Let us first suppose that a point $r$ exists such that $r$ is non-collinear with every two of the points $p_{1}, p_{2}, q_{1}, q_{2}$. In the first case, collineations $\tau_{3}$ and $\tau_{4}$ exist such that $\tau_{3}\left(p_{1}, p_{2}, r, q_{1}\right)=q_{1}, p_{2}, r, p_{1}$ and $\tau_{4}\left(q_{1}, p_{2}, r, q_{2}\right)=q_{1}, q_{2}, r, p_{2}$, and therefore $\tau_{4} \tau_{3}$ is the required collineation. In the second case, collineations $\tau_{5}$ and $\tau_{6}$ exist such that $\tau_{5}\left(p_{1}, p_{2}, r, q_{2}\right)$ $=p_{1}, q_{2}, r, p_{2}$ and $\tau_{6}\left(p_{1}, q_{2}, r, q_{1}\right)=q_{1}, q_{2}, r, p_{1}$ and in this case $\tau_{6} \tau_{5}$ is a collineation that transforms $p_{1}, p_{2}$ into $q_{1}, q_{2}$ respectively.

If no such point $r$ exists then every point is contained in some one of the one-spaces determined by two of the four points $p_{1}, p_{2}, q_{1}, q_{2}$. Let us consider the case where $p_{1}$ and $p_{2}$ are on no line and where $p_{1}$ is collinear with $q_{1} q_{2}$ and let us further suppose that $p_{1}$ is distinct from $q_{1}$ and from $q_{2}$. All points are in the two-space or plane determined by the line $q_{1} q_{2}$ and the point $p_{2}$ is not in the line $q_{1} q_{2}$. If $n=2$ then by Postulate $\mathrm{V}$ a collineation not the identity exists having $p_{1}, q_{1}, q_{2}$ as invariant points and there must therefore be at least one additional point $r_{1}$ on the one-space $q_{1} p_{2}$ or one additional point $s_{1}$ on the one-space $q_{2} p_{2}$. If $r_{1}$ exists then collineations $\tau_{7}$ and $\tau_{8}$ exist such that $\tau_{7}\left(p_{1}, p_{2}, r_{1}, q_{2}\right)$ $=p_{1}, q_{2}, r_{1}, p_{2}$ and $\tau_{8}\left(q_{1}, q_{2}, p_{1}\right)=p_{1}, q_{2}, q_{1}$. If $s_{1}$ exists we have collineations $\tau_{9}$ and $\tau_{10}$ such that $\tau_{9}\left(p_{1}, p_{2}, s_{1}, q_{1}\right)=p_{1}, q_{1}, s_{1}, p_{2}$ and

$$
\tau_{10}\left(q_{1}, q_{2}, p_{1}\right)=q_{2}, p_{1}, q_{1} \text {. }
$$

Hence $\tau_{8} \tau_{7}$ or $\tau_{10} \tau_{9}$ will be the required collineation according as $r_{1}$ or $s_{1}$ exists. If $n>2$ there are by Postulate I at least $n+2>4$ distinct points; hence the additional point $r_{1}$ or $s_{1}$ exists as before, and the argument is completed as in the case $n=2$.

Let us now suppose that $p_{1}$ coincides with $q_{1}$. Since $n \geqq 2$, at least four 
distinct points exist. Let us assume $q_{3}$, distinct from $q_{1}$ and $q_{2}$, exists on the one-space $q_{1} q_{2}$. A collineation distinct from the identity exists leaving invariant $q_{1}, q_{2}, q_{3}$ if $n=2$ and, if $n>2, q_{1}, q_{2}, q_{3}$, and $n-2$ other points; therefore a point $r_{1}$ distinct from $q_{2}, p_{2}$ must lie on the one-space $q_{2} p_{2}$. If the existence of $r_{1}$ had been assumed, then we could have proven in a similar manner that $q_{3}$ existed and since one or the other must exist, both exist. Since the four points $p_{1}=q_{1}, p_{2}, r_{1}, q_{3}$ are such that no three are collinear a collineation $\tau_{11}$ exists such that $\tau_{11}\left(p_{1}, p_{2}, r_{1}, q_{3}\right)=p_{1}, q_{3}, r_{1}, p_{2}$ and a collineation $\tau_{12}$ exists such that $\tau_{12}\left(p_{1}, q_{2}, q_{3}\right)=q_{1}, q_{3}, q_{2}$. The collineation $\tau_{12} \tau_{11}$ is the collineation that transforms $p_{1} p_{2}$ into $q_{1} q_{2}$. For all the other possible cases the proofs are very similar to the preceding and therefore need not be repeated.

Theorem 6. A line exists.

If all linear sets contained but two points there would be but $k+1$ points in a $k$-space. This would make Postulates I and VI contradictory and therefore at least one linear set contains more than two points and hence a line exists.

Theorem 7. Every collineation transforms lines into lines.

Let $\tau_{1}$ be any collineation that transforms the line $P=[p]$ into a set of points $Q=[q]$. We are to prove that the set of points $Q$ constitute a linear set. Let $\tau_{2}$ be any collineation that leaves two of the $q$ 's, say $q_{1}$ and $q_{2}$ invariant. The points $q_{1}$ and $q_{2}$ are the transforms under $\tau_{1}$ of two points of $P$ which we will call $p_{1}$ and $p_{2}$. By Theorem 5 there is a collineation $\tau_{3}$ that transforms $q_{1}, q_{2}$ into $p_{1}, p_{2}$. Then $\tau_{3} \tau_{2} \tau_{1}(P)=P$ since the points $p_{1}$ and $p_{2}$ of $P$ are left invariant. For the same reason $\tau_{3} \tau_{1}(P)=P$. Therefore $\tau_{3}(Q)=P$, that is to say every $p$ is the transform under $\tau_{3}$ of some $q$ and that for every $q \tau_{3}(q)$ is a $p$. But $\tau_{3} \tau_{2}(Q)=P$ therefore $\tau_{2}(Q)=Q$. Hence any collineation that leaves two points of $Q$ fixed leaves $Q$ invariant.

Let $\tau_{4}$ be any collineation that leaves three of the $q$ 's invariant, say $q_{1}, q_{2}, q_{3}$, where $q_{1}, q_{2}, q_{3}$ are the transforms under $\tau_{1}$ of $p_{1}, p_{2}, p_{3}$ respectively. $\tau_{3} \tau_{4} \tau_{1}[P]=P$ since $p_{1}$ and $p_{2}$ are left invariant. $\tau_{3} \tau_{4} \tau_{1}\left(p_{1}, p_{2}, p_{3}\right)$ $=p_{1}, p_{2}, p_{3}^{\prime}$. Since $p_{1}, p_{2}, p_{3}$ and $p_{1}, p_{2}, p_{3}^{\prime}$ are sets of collinear points a collineation $\tau_{5}$ exists such that $\tau_{5}\left(p_{1}, p_{2}, p_{3}\right)=p_{1}, p_{2}, p_{3}$. Then

and

$$
\tau_{5} \tau_{3} \tau_{4} \tau_{1}\left(p_{1}, p_{2}, p_{3}\right)=p_{1}, p_{2}, p_{3}
$$

$$
\tau_{5} \tau_{3} \tau_{1}\left(p_{1}, p_{2}, p_{3}\right)=p_{1}, p_{2}, p_{3} .
$$

Therefore the collineations $\tau_{5} \tau_{3} \tau_{1}$ and $\tau_{5} \tau_{3} \tau_{4} \tau_{1}$ leave every point of $P$ invariant. If $\tau_{1}$ transforms $p_{i}$ into $q_{i}, \tau_{5} \tau_{3}$ must transform $q_{i}$ into $p_{i}$ and hence $\tau_{4}$ must leave every point of $Q$ invariant. We have shown that properties $(a)$ and $(b)$ of a linear set hold for $Q$ and since $P$ was a line (containing at least three points) $Q$ is a line. 
Theorem 8. If $p_{1}$ and $p_{2}$ are distinct points, there is at least one line containing both $p_{1}$ and $p_{2}$.

We know that at least one line exists from Theorem 6. That line has two points $q_{1}$ and $q_{2}$. By Theorem 5 a collineation $\tau$ exists transforming $q_{1}, q_{2}$ into $p_{1}, p_{2}$ respectively. The line containing $q_{1}, q_{2}$ is transformed by $\tau$ into a line which contains $p_{1}, p_{2}$.

ThEOREM 9. Every collineation transforms a $k$-space into a $k$-space.

Theorem 10. All points are not on the same line.

THEOREM 11. If $p_{1}, p_{2}, p_{3}, p_{4}, p_{5}$ are five distinct points such that $p_{1}, p_{2}, p_{3}$ are non-collinear, $p_{1}, p_{2}, p_{4}$ are collinear and $p_{1}, p_{3}, p_{5}$ are collinear, then there exists a point $p_{6}$ such that $p_{2}, p_{3}, p_{6}$ are collinear and $p_{4}, p_{5}, p_{6}$ are collinear.*

No three of the points $p_{2}, p_{3}, p_{4}, p_{5}$ are collinear for if they were $p_{1}, p_{2}, p_{3}$ would be collinear. By Theorem 4 a collineation $\tau$ exists such that

$$
\tau\left(p_{2}, p_{3}, p_{4}, p_{5}\right)=p_{2}, p_{4}, p_{3}, p_{5}
$$

The collineation $\tau$ transforms the lines $p_{3} p_{5}$ and $p_{2} p_{4}$ into the lines $p_{4} p_{5}$ and $p_{2} p_{3}$ respectively. The point $p_{1}$ common to the lines $p_{3} p_{5}$ and $p_{2} p_{4}$ will therefore be transformed into a point $p_{6}$ common to the lines $p_{4} p_{5}$ and $p_{2} p_{3}$.

We have already proven as theorems the Veblen-Young postulates $A_{1}, A_{2}$, $A_{3}, E_{0}, E_{1}$, and $E_{2}$. The postulate $A_{1}$ is our Theorem 8; $A_{2}$ is our Theorem $1 ; A_{3}$ (if $p_{1}, p_{2}, p_{3}$ are points not all on the same line and $p_{4}$ and $p_{5}\left(p_{4} \neq\right.$ $p_{5}$ ) are points such that $p_{1}, p_{2}, p_{4}$ are on a line and $p_{1}, p_{3}, p_{5}$ are on a line, there is a point $p_{6}$ such that $p_{2}, p_{3}, p_{6}$ are on a line and $p_{4}, p_{5}, p_{6}$ are on a line) is in content equivalent to Theorem $11 ; E_{0}$ (there are at least three points on every line) is true from definition of line; $E_{1}$ (there exists at least one line) is Theorem 6; and $E_{2}$ is Theorem 9 . We therefore know that our line satisfies the six preceding postulates that Veblen and Young lay down for their undefined line, hence all theorems that they derive from the six assumptions listed will hold in our geometry. One such theorem is:

Theorem 12. Let the $k$-space $P_{k}$ be defined by the point $p_{0}$ and the $(k-1)$ space $P_{k-1}$, then

(a) There is a k-space on any $k+1$ independent points.

(b) Every line on two points of $P_{k}$ has one point in common with $P_{k-1}$ and is in $P_{k}$.

(c) Every $P_{g}(g<k)$ on $g+1$ independent points of $P_{k}$ is in $P_{k}$.

* Theorem 11 is essentially equivalent to the Veblen-Young postulate $A_{3}$ : If $p_{1}, p_{2}, p_{8}$ are points not all on the same line and $p_{4}$ and $p_{5}\left(p_{4} \neq p_{5}\right)$ are points such that $p_{1}, p_{2}, p_{4}$ are on a line and $p_{1}, p_{3}, p_{5}$ are on a line, there is a point $p_{6}$ such that $p_{2}, p_{3}, p_{6}$ are on a line and $p_{4}, p_{5}, p_{6}$ are on a line. The form of statement for Theorem 11 was suggested by Professor E. H. Moore as a substitute for $A_{3}$ since the latter is redundant in that it includes the obvious cases where $p_{4}$ is coincident with $p_{1}$ or $p_{2}$ or where $p_{5}$ is coincident with $p_{1}$ or $p_{3}$. 
(d) Every $P_{g}(g<k)$ on $g+1$ independent points of $P_{k}$ has a $P_{g-1}$ in common with $P_{k-1}$ provided all $g+1$ points are not in $P_{k-1}$.

(e) Every line $P_{1}$ on two points of $P_{k}$ has one point in common with every $P_{k-1}$ in $P_{k}$.

(f) If $q_{0}$ and $Q_{k-1}\left(q_{0}\right.$ not in $\left.Q_{k-1}\right)$ are any point and any $(k-1)$-space respectively of the $k$-space determined by $p_{0}$ and $P_{k-1}$, the latter space is the same as that determined by $q_{0}$ and $Q_{k-1}$.

Another important theorem that Veblen and Young prove is:

Theorem 13. On $k+1$ independent points there is one and but one $k$-space.

Theorem 14. If $k+1$ points of a $P_{k-1}$, such that every $k$ are independent, are left invariant by a collineation $\tau$ then $\tau$ leaves every point of $P_{k-1}$ invariant.

This theorem clearly is true for a line or $P_{1}$. Let us assume that the theorem is true for a $P_{g-1}$. In a $P_{g}$ if every $g+1$ of $g+2$ points are to be independent, then if $p_{1}, \cdots, p_{g}$ determine a $P_{g-1}, p_{g+1}$ and $p_{g+2}$ cannot lie in $P_{g-1}$, nor can the line $p_{g+1} p_{g+2}$ contain any of the points $p_{1}, \cdots, p_{g}$. The line $p_{g+1} p_{g+2}$ has a single point $p_{0}$ in common with $P_{g-1}$ by Theorem $12(e)$. When the $g+2$ points are left invariant $p_{0}$, being the intersection of $P_{g-1}$ and $p_{g+1} p_{g+2}$, is left invariant. The line $P_{g-1}$ and the line $p_{g+1} p_{g+2}$ are each therefore left identically invariant. Let $p$ be any point in $P_{g}$ not in $P_{g-1}$ nor on the line $p_{g+1} p_{g+2}$. The lines $p p_{g+1}$ and $p p_{g+2}$ each meet the $P_{g-1}$ by Theorem $12(e)$. When the given $g+2$ points are left invariant, these lines and consequently their intersection is left invariant. The theorem being true for a $g$-space if true for a $(g-1)$-space and holding for a one-space is therefore true for a $k$-space.

TheOREm 15. All points are not on the same $k$-space if $k<n$.

It can easily be shown that every $k$-space has $k+2$ points such that every $k+1$ are independent. If these $k+2$ points and $n-k-1$ other points, which exist by Postulate I, are not in the $k$-space the theorem is true. If these $n+1$ points are in the $k$-space then by Postulate $\mathrm{V}$ a collineation distinct from the identity exists leaving these $n+1$ points invariant and therefore by Theorem 14 that collineation leaves the $k$-space identically invariant. Hence not all points can be in the $k$-space.

Theorem 16. There exist $n+2$ points such that every $n+1$ are independent. The definitions of perspectivity, projectivity, etc., can now be given exactly as Veblen and Young give them. We will now proceed to identify what we call a collineation with what they call a projective collineation. To do this we will first prove:

Theorem 17. Every central perspective correspondence between points of two lines can be secured by a collineation.

Let the perspectivity be defined by $p_{1}, p_{2}$ having as their correspondents $q_{1}, q_{2}$. We have by Theorem 4 , since no three of $p_{1}, p_{2}, q_{1}, q_{2}$ are collinear, 
that the two collineations $\tau_{1}$ and $\tau_{2}$ exist such that

and

$$
\tau_{1}\left(p_{1}, p_{2}, q_{1}, q_{2}\right)=p_{1}, q_{2}, q_{1}, p_{2}
$$

$$
\tau_{2}\left(p_{1}, q_{2}, q_{1}, p_{2}\right)=q_{1}, q_{2}, p_{1}, p_{2},
$$

therefore $\tau=\tau_{2} \tau_{1}$ exists by Postulate II and is such that $\tau\left(p_{1}, p_{2}, q_{1}, q_{2}\right)$ $=q_{1}, q_{2}, p_{1}, p_{2}$. The lines $p_{1} q_{1}$ and $p_{2} q_{2}$ will be left invariant by $\tau$ and so will their point of intersection $o$. The lines $p_{1} p_{2}$ and $q_{1} q_{2}$ are interchanged by $\tau$ as well as the lines $p_{1} q_{2}$ and $p_{2} q_{1}$. Let the intersection of $p_{1} p_{2}$ and $q_{1} q_{2}$ be $r_{3}$ and of $p_{1} q_{2}$ and $p_{2} q_{1}$ be $r_{4}$. The collineation $\tau$ must leave $r_{3}$ and $r_{4}$ invariant. There are two cases possible, ${ }^{*}$ the first where $o, r_{3}$, and $r_{4}$ are non-collinear and the second where these three points are collinear. Let us first consider case 1 . The line $r_{3} r_{4}$ meets the lines $p_{1} q_{1}$ and $p_{2} q_{2}$ in points $r_{1}$ and $r_{2}$. The collineation $\tau$ leaves $r_{1}, r_{2}, r_{3}$, and $r_{4}$ invariant; hence every point of the line $r_{3}, r_{4}$ is left invariant by $\tau$. Let $p$ be any point on the line $p_{1} p_{2}$ and call $r$ the point of intersection of the line op with the line $r_{1} r_{2}$. The line $o p$ is left invariant by $\tau$ since that collineation leaves two of its points, $o$ and $r$, invariant. Since the line $p_{1} p_{2}$ is transformed into the line $q_{1} q_{2}$ by $\tau, p$ is transformed by $\tau$ into the intersection of the lines op and $q_{1} q_{2}$ which we will call $q$.

For the second case, where $o, r_{3}$, and $r_{4}$ are collinear, the points $o$ and $r$ are coincident. The collineation $\tau^{2}=\tau \tau$ leaves $p_{1}, p_{2}$, and $r_{3}$ invariant and therefore leaves every point of $p_{1} p_{2}$ invariant. Hence if $q$ denotes the point of $q_{1} q_{2}$ into which $\tau$ transforms a point $p$ of $p_{1} p_{2}$ then $\tau$ must transform $q$ into $p$. Hence the line $p q$ is left invariant by $\tau$. Since $o, r_{3}$, and $r_{4}$ are collinear the lines $p_{1} q_{1}$, $p_{2} q_{2}$, and $r_{3} r_{4}$ are concurrent at $o$. If the line $p q$ did not pass through $o$ it would intersect the three lines $p_{1} q_{1}, p_{2} q_{2}$, and $r_{3} r_{4}$ at three distinct points. But this would make the line $p q$ identically invariant under the collineation $\tau$ and hence $p$ would be invariant and coincide with $q$, but this is possible only if $p$ is $r_{3}$ and we assumed that $p$ was any point on the line $p_{1} p_{2}$ therefore $p q$ passes through $o$. Therefore the collineation $\tau$ makes correspond to the points of the line $(p)$ the points of line $(q)$ perspective to $(p)$ with center of perspectivity 0 .

THEOREM 18. If a projective correspondence exists between the points of two lines, then a collineation exists that transforms the points of the first line into the projectively corresponding points of the second line.

A projective correspondence between the points of two lines is the resultant of a sequence of central perspectivities. By Theorem 16, each central perspective correspondence has associated with it a collineation. The resultant of the sequence of collineations corresponding to the sequence of perspectivities that define the projectivity is the required collineation.

* If Postulate VIII were assumed, the first case only would arise. 
Theorem 19. If a projectivity leaves each of three distinct points of a line invariant it leaves every point of the line invariant.

Theorem 20. If $P$ is a $P_{n}$ all points are in $P$.

This theorem follows readily from Postulate VI and Theorem 12.

In addition to the Veblen-Young postulates $A_{1}, A_{2}, A_{3}, E_{0}$, and $E_{1}$ we have proven $E_{n}$, which is Theorem $15, E_{n}^{\prime}$, which is Theorem 19 , and $P$, which is Theorem 18. We have therefore proven all of the postulates that Veblen and Young assume for general projective geometry of $n$-space and consequently all the theorems that can be derived from their postulates hold in our geometry.

We can now prove

Theorem 21. Every collineation is a projective collineation.

Let $\tau$ be any collineation. From Theorem 20 we know that there exist $n+2$ points such that every $n+1$ are independent. The collineation $\tau$ will transform these $n+2$ points which we will call $p_{1}, p_{2}, \cdots, p_{n+2}$ into $p_{1}^{\prime}, p_{2}^{\prime}$, $\cdots, p_{n+2}^{\prime}$ respectively. There exists a projective collineation $\pi$ (from the Veblen-Young geometry which is at our disposal) such that $\pi\left(p_{1}, p_{2}, \cdots, p_{n+2}\right)$ $=p_{1}^{\prime}, p_{2}^{\prime}, \cdots, p_{n+2}^{\prime}$. The points $p_{1}, p_{2}, \cdots, p_{n}$ determine an $(n-1)$-space which will meet the line $p_{n+1} p_{n+2}$ in a point $p_{0}$. Let $p_{0}^{\prime}$ denote the intersection of the $(n-1)$-space determined by $p_{1}^{\prime}, p_{2}^{\prime}, \cdots, p_{n}^{\prime}$ with the line $p_{n+1}^{\prime} p_{n+2}^{\prime}$. This point, $p_{0}^{\prime}$, will correspond to $p_{0}$ both by transformation $\tau$ and transformation $\pi$. The line $p_{n+1} p_{n+2}$ having three of its points $p_{n+1}, p_{n+2}$, and $p_{0}$ transformed into $p_{n+1}^{\prime}, p_{n+2}^{\prime}$, and $p_{0}$ both by $\tau$ and $\pi$, will have every one of its points transformed into the same corresponding point of $p_{n+1}^{\prime} p_{n+2}^{\prime}$ both by $\tau$ and $\pi$. This is true for every line $p_{i} p_{j}(i, j=1,2,3, \cdots, n+2 ; i \neq j)$. Every plane $p_{i} p_{j} p_{k}$ will go into the plane $p_{i}^{\prime} p_{j}^{\prime} p_{k}^{\prime}$ and every point in the first plane will have the same correspondent in the second plane both by $\tau$ and $\pi$. Let $p_{i j k}$ be any point in the plane $p_{i} p_{j} p_{k}$. Draw the lines $p_{i} p_{i j k}$ and $p_{j} p_{i j k}$. These lines will intersect the lines $p_{j} p_{k}$ and $p_{i} p_{k}$ in points that we can call $p_{j k}$ and $p_{i k}$. Transformations $\tau$ and $\pi$ will transform $p_{i}, p_{j}, p_{j k}$, and $p_{i k}$ into the same points, that is into $p_{i}^{\prime}, p_{j}^{\prime}, p_{j k}^{\prime}$, and $p_{i k}^{\prime}$. To $p_{i j k}$ will correspond the intersection of $p_{i}^{\prime} p_{j k}^{\prime}$ with $p_{j}^{\prime} p_{i k}^{\prime}$ by either $\tau$ or $\pi$. By continuing this process we can prove that to any point $p$ there corresponds by either $\tau$ or $\pi$ the same point $p^{\prime}$. Hence $\tau$ and $\pi$ are identical.

If we desire the set of collineations from which we start to be the group of all projective collineations we add Postulate VII to the postulates we have used and we have the theorem.

Theorem 22. Every projective collineation is a collineation. 


\section{INDEPENDENCE EXAMPLES, $n \geq 3$}

In the following a Roman numeral preceded by a minus sign denotes an example of a system in which the postulate denoted by that numeral is false but all the other postulates of the set I-VIII are true.*

- I. Let the class of points consist of a single element and the class of collineations of the identity transformation.

- II. Case $1, n$ odd. Let the class of points consist of $2(n+1)$ elements in $(n+1) / 2$ sets of four. Let the collineations be all the transformations that permute the points of each set amongst themselves, the identity transformation excepted, together with all the transformations that permute the points of each of all but two sets amongst themselves but leaves one point of each of the two remaining sets invariant and transforms the other three points of each of these sets into the remaining three points of the other set. Each set is a line and there are no other lines.

Case 2, $n$ even. Let the class of points consist of $2 n+1$ points in $n / 2$ sets of four and one single point. Let the collineations be transformations on the $n / 2$ sets like those of Case 1 leaving the extra point invariant together with the transformations that permute the points of each of all but one of the sets amongst themselves, interchange the single point with one of the points of the remaining set and leaves none of the three other points of that set invariant. Each set is again a line and there are no other lines.

- III. Let the class of points be $2(n+1)$ elements in $n+1$ sets of two. The collineations are the $2^{n+1}$ transformations on the points leaving each pair invariant. The lines are the $n(n+1) / 2$ tetrads of points each consisting of two pairs.

- IV. Case $1, n$ odd. Let the class of points consist of $2(n+1)$ elements in $(n+1) / 2$ sets of four. Let the class of collineations be all the transformations that permute the points of each set or interchange the sets. Each set is a line.

Case 2, $n$ even. Let the class of points consist of $2 n+1$ elements in $n / 2$ sets of four and one single point. Let the collineations be like those of case 1 on the sets and leave the extra point invariant. Each set is a line.

$-\mathrm{V}$. Ordinary projective geometry of $(n-1)$-space.

- VI. Ordinary projective geometry of $(n+1)$-space.

- VII. Let the class of points be the class of all sets of $n+1$ rational numbers $\left(X_{1}, X_{2}, \cdots, X_{n+1}\right)$, the set $(0,0, \cdots, 0)$ excepted. The sets $\left(X_{1}, X_{2}, \cdots, X_{n+1}\right)$ and $\left(K X_{1}, K X_{2}, \cdots, K X_{n+1}\right)$ are understood to be equivalent for all rational values of $k$ distinct from zero. Let the collineations be the class of all linear homogeneous transformations on $n+1$ variables,

*In - I, Postulates III-VIII are satisfied vacuously. The same is true of Postulates VII and VIII in - I and - III and of Postulate VII in - IV. 
having rational coefficients and whose determinants of transformation are $(n+1)$ th powers of rational numbers not zero.

- VIII. The finite projective geometry of $n$-space having three points on a line.*

* For finite projective geometries see O. Veblen and W. H. Bussey, Finite projective geometries, these Tra n s a c t i o s s, vol. 7 (1906), pp 241-259. 